\title{
Energy cost associated with moving platforms
}

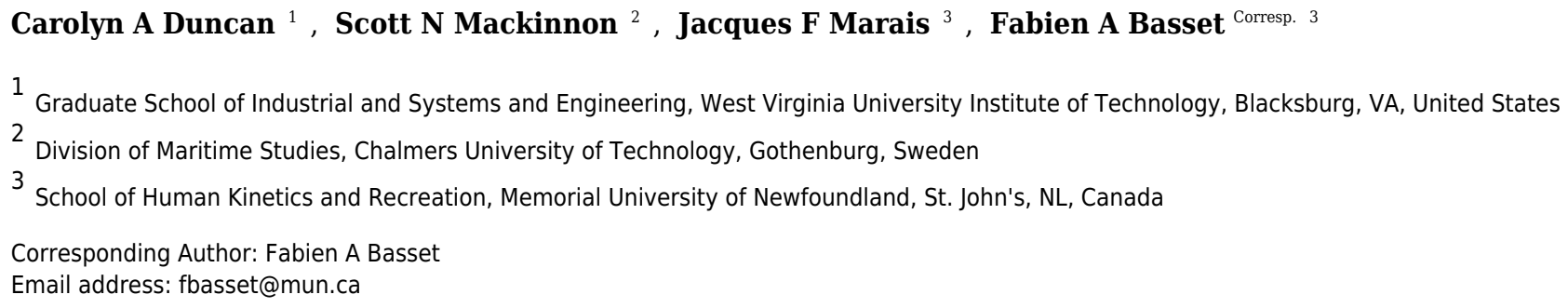

Background: Previous research suggests motion induced fatigue (MIF) contributes to significant performance degradation and is likely related to a higher incidence of accidents and injuries. However, the exact effect of continuous multidirectional platform perturbations on energy cost with experienced personnel on boats and other seafaring vessels remains unknown. Objective: The objective of this experiment was to measure the metabolic energy costs (EC) associated with maintaining postural stability in a motionrich environment. Methods: Twenty volunteer participants, who were free of any musculoskeletal or balance disorders, performed three tasks while immersed in a moving environment that varied motion profiles similar to those experienced by workers on a midsize commercial fishing vessel [static platform (baseline), low and high motions]. Cardiorespiratory parameters were collected using an indirect calorimetric system that continuously measured breath-by-breath samples. Heart rate was recoded using a wireless heart monitor. Results: Results indicate a systematic increase in metabolic costs associated with increased platform motions. The increases were most pronounced during the standing and lifting activities and were $50 \%$ greater during the high motion condition when compared to no motion. Increased heart rates were also observed. Discussion: Platform motions have a significant impact on metabolic costs that are both task and magnitude of motion dependent. Practitioners must take into consideration the influence of motion rich environments upon the systematic accumulation of operator fatigue. 
2

3

4

5

6

7

8

9

10

11

12

13

14

15

16

17

18

19

20

21

22 Corresponding Author:

23 Fabien A. Basset, PhD

24 Address: School of Human Kinetics and Recreation

25 Memorial University of Newfoundland

26 St. John's, NL, CANADA, A1C 5S7

27 Telephone: (709) 864-6132

28 Email: fbasset@,mun.ca

\section{ENERGY COSTS ASSOCIATED WITH MOVING PLATFORMS}

Carolyn A. Duncan ${ }^{1}$, Scott N. MacKinnon², Jacques F. Marais ${ }^{3}$, Fabian A. Basset ${ }^{3}$

${ }^{1}$ Graduate School of Industrial and Systems and Engineering, Virginia Tech, Blacksburg, VA, USA

${ }^{2}$ Division of Maritime Studies, Chalmers University of Technology, Gothenburg, Sweden

${ }^{3}$ School of Human Kinetics and Recreation, Memorial University of Newfoundland, St. John's, NL, Canada 


\section{Abstract}

31

Background: Previous research suggests motion induced fatigue contributes to significant

33 performance degradation and is likely related to a higher incidence of accidents and injuries.

34 However, the exact effect of continuous multidirectional platform perturbations on energy cost

35 with experienced personnel on boats and other seafaring vessels remains unknown.

36 Objective: The objective of this experiment was to measure the metabolic energy costs associated 37 with maintaining postural stability in a motion-rich environment.

38 Methods: Twenty volunteer participants, who were free of any musculoskeletal or balance

39 disorders, performed three tasks while immersed in a moving environment that varied motion

40 profiles similar to those experienced by workers on a mid-size commercial fishing vessel (static

41 platform (baseline), low and high motions). Measures of ventilation were collected using a portable

42 metabolic system that continuously measured breath-by-breath samples and heart rate using a

43 wireless heart monitor.

44 Results: Results indicate a systematic increase in metabolic costs associated with increased 45 platform motions. The increases were most pronounced during the standing and lifting activities 46 and were $50 \%$ greater during the high motion condition when compared to no motion. Increased 47 heart rates were also observed.

48 Discussion: Platform motions have a significant impact on metabolic costs that are both task and 49 magnitude of motion dependent. Practitioners must take into consideration the influence of motion 50 rich environments upon the systematic accumulation of operator fatigue. 


\section{ABBREVIATIONS:}

54 BoS: base of support

55 CoM: centre of mass

56 HM: high motion

57 LM: low motion

58 MII: motion induced interruption

59 MMH: manual materials handling

60 NM: no motion

61 RER: respiratory exchange ratio

62 RMR: resting metabolic rate

63

64 


\section{Introduction}

66 Motion-rich environments may have effects on the human body that can adversely impact

67 biomechanical, physiological and psychological aspects of vocational performance (Crossland

68 and Lloyd, 1993; Crossland, 1994; Wertheim, 1998; Duncan et al., 2010; Duncan et al., 2012).

69 More specifically, platform motions can negatively impact upon an operator's ability to manage

70 command, control and communication systems, carry out navigational tasks, perform normal

71 ship operation and maintenance functions and prepare food in maritime industries that involve

72 moving platforms including but not limited to coast guard, maritime shipping and the offshore

73 petroleum industry (Dobie, 2003). While the strenuous and dangerous nature of many offshore

74 occupations is obvious, wave induced platform motions are likely responsible for accidents and

75 injuries associated with reduced postural stability and increased work-related energy costs

76 (Wertheim, 1998).

Effects on performance have been related to magnitude and frequency of the platform motions

79 (Colwell, 1989; Bles et al, 1998; Wertheim, 1998; MacKinnon et al, 2011 Duncan et al., 2014).

80 Continuous exposure to motion-rich environments makes performing tasks more difficult

81 because of the continuous postural adjustments needed to maintain balance (Duncan et al, 2007).

82 These required postural adjustments will add to the energy cost compared to work done in a

83 stable environment (Baitis et al, 1995; Wertheim, 1998). This increased energy cost may be

84 related to motion induced fatigue (MIF). MIF has been shown to increase injury occurrences and

85 decrease productivity in offshore workers (Colwell, 1989; Haward et al, 2009). To the authors'

86 knowledge current research on MIF fatigue is limited. It has been suggested MIF can be broadly

87 characterized into two categories: fatigue due to loss or poor quality of sleep and the added 
88 energy costs associated with doing work in a moving environment. Unlike motion induced

89 sickness, crew members are unable to habituate to the effects of MIF, likely resulting in

90 accumulative effects leading to crew ineffectiveness such as lack or loss of situation awareness

91 and errors in judgment (Stevens and Parsons, 2002). Accumulated fatigue can also impact upon

92 the ability and quality of performing manual materials handling (MMH) activities in a safe and

93 efficient manner. A better understanding of the impact of increased energy cost in moving

94 environments may provide insight into the design and execution of tasks performed in maritime

95 environments and lead to a reduction of risk of errors and injuries and improvements in safety

96 and performance.

98 Accidents and injuries at sea often identify operator fatigue as the contributing cause, however,

99 little research to date has attempted to quantify the relationship between platform motion

100 magnitude and operator energy cost. Wertheim (1998) reported a series of earlier studies that

101 measured energy cost via indirect calorimetry technique in a moving environment. All of these

102 were done under controlled conditions within a ship motion simulator facility. A variety of

103 activities, including standing, treadmill running, and crate stacking were examined. Results

104 showed increases in oxygen uptake of 7\% compared to no motion condition during the simulated

105 ship movements although the increases were less than expected. Whether self-reported or

106 observed by investigators, participants consistently reported being "severely" fatigued following

107 testing. While a holistic approach to studying any problem is desirable, it is recognized that

108 focused research on the mechanisms underlying MIF physiological responses is required. By

109 addressing this knowledge gap, it is expected that such research will improve upon the 
110 understanding of the direct and indirect effects of MIF on human performance in moving

111 environments.

112 The purpose of this study was to quantify the additional energy required by persons performing

113 common tasks in simulated maritime environments as compared to stable (i.e. land)

114 environments. It is hypothesized that the continuous multi-directional perturbations will

115 significantly increase the energy costs associated with performing sitting, standing and manual

116 materials handling tasks.

117

118 Materials \& Methods

119 Participants

120 Twenty (10 males, 10 females) (age: $23.4 \pm 2.0$ years; mass: $74.5 \pm 13.1 \mathrm{~kg}$; stature: $172 \pm 7.0 \mathrm{~cm}$;

121 BMI: $25.1 \pm 4.8 \mathrm{~kg} \mathrm{~m}^{-2}$ ) participants were recruited for this study. Participants reported no

122 susceptibility to motion sickness and were free of balance disorders and musculoskeletal diseases

123 or injuries. Due to the influence of experience on habituation and postural control (Duncan et al.,

124 In Press) only individuals with no experience working in maritime environments were eligible

125 for this study. Ethical approval for this experiment was given by the Memorial University of

126 Newfoundland's Human Investigations Committee. All participants have signed the consent

127 form prior to undergoing any physical tests.

128

129 Procedures

130 Participants performed sitting, standing and lifting/lowering tasks while being exposed to three

131 motion conditions (no motion, low motion, high motion). The experimental protocol was

132 completed over three sessions with at least twenty-four hours between each session to ensure for

133 sufficient recovery and to reduce the effects of accumulative fatigue. Each session required the 
134 participant to perform a task (i.e. quiet sitting on a chair, standing in an upright position, or

135 lifting and lowering a load) for each of the three motion conditions. Each session began with

136 quiet sitting while resting metabolic rate (RMR) was collected. Exposure to each motion

137 condition lasted ten minutes with a minimum of a five-minute rest period between conditions.

138 While every attempt was made to randomize the task order presentation of the motion, one

139 concession was made: the no motion trial was always presented to the participant first. The no

140 motion trial was considered to be a baseline condition from which all other data collection

141 sessions could be compared or perhaps could serve as a normalizing value. Subsequently, the

142 low or high motion trial was randomly presented so all participants got both high and low motion

143 conditions (Figure 1). Participants were instructed to refrain from smoking and any physical

144 activity or consume food or drink (except water) three hours prior to testing (Compher et al, 145 2006).

Insert Figure 1 about here

149 Experimental Tasks

150 Participants required to sit on a chair (seat width: $0.48 \mathrm{~m}$; seat depth: $0.48 \mathrm{~m}$; seat height: $0.42 \mathrm{~m}$;

151 armrest height: $0.64 \mathrm{~m}$; backrest height: $0.77 \mathrm{~m}$ ) firmly situated to the platform with front legs in

152 the middle of the platform facing the "bow" (front) direction for all trials. Throughout the task

153 the participants were instructed to keep their torsos against the backrest, arms on the arm rests, 154 and legs uncrossed with feet placed flat on the ground. 
156 During standing trials participants remained in the middle of the motion platform in an erect

157 standing position with arms along their sides, feet approximately shoulder width apart, weight

158 evenly distributed between their feet and facing the bow of the platform (Figure 2). Stepping was

159 permitted to correct for balance interruptions during the motion trials. Upon stepping participants

160 were instructed to return to the original foot positions as soon as balance has been regained.

161

162

163

164

165

166

167

168

169

170

171

172

173

174

175

176

177

Insert Figure 3 about here

Insert Figure 2 about here lowering technique.

178

A bimanual sagittal plane lifting/lowering task required participants to lift and lower a $5.0 \mathrm{~kg}$ load (length: $0.327 \mathrm{~m}$; width: $0.327 \mathrm{~m}$, height: $0.270 \mathrm{~m}$ ) directly to and from a table (width: 1.54 $\mathrm{m}$, depth: $0.52 \mathrm{~m}$; height from floor: $0.72 \mathrm{~m}$ ) securely situated $0.60 \mathrm{~m}$ directly in front of the participant facing the bow direction (Figure 3). Load size and lift dimensions were determined from the revised NIOSH lifting equation for safe lifting (Waters et al., 1993). Throughout the task participants were required to keep their feet shoulder width apart and parallel to the table. Lifts and lowers were separated by ten second intervals. The start of each lift or lower were initiated via audible signals and were performed consecutively at a rate of three lifts per minute and three lowers per minute resulting in a cumulative task rate of six manipulations per minute for a total of $60 \mathrm{MMH}$ events for each condition. Participant used their own preferred lifting and 
179

180

181

182

183

184

185

186

187

188

189

190

191

192

193

194

195

196

197

198

199

200

201

202

97

98

\section{Experimental Apparati}

All motion conditions were performed on a Moog 6DOF2000E electric motion platform. The platform consisted of a 2 by $2 \mathrm{~m}$ metal platform with $1.02 \mathrm{~m}$ high railings along the perimeter. A canopy enclosure eliminated external horizontal and vertical cues from the participant's field of vision. Motion conditions varied in amplitude and frequency and were derived from captured wave induced ship motions using linear wave theory that allowed for the profile to vary in magnitude (Lloyd, 1993). Five degrees of freedom were used (roll, pitch, heave, surge, sway) (Figure 4). Linear equations used to develop all motion profiles are detailed (Equations 1-5). The low motion (LM) profile reflected a $10 \%$ increase in motion frequency relative to the original vessel from which the motion profiles were recorded and the high motion (HM) profile was characterized by a $15 \%$ increase in frequency and a $265 \%$ increase in amplitude relative to the seagoing motion profile (Table 1). Statistical analysis of differences between these conditions, performed using students' t-tests, confirm that the RMS of the LM and HM conditions were significantly difference in pitch and roll directions $(p<0.001)$. These conditions were compared to a stable, no motion condition (NM).

(1)

Insert Figure 4 about here

$\operatorname{Roll}(\mathrm{x})(\mathrm{deg} / \mathrm{s} / \mathrm{s})=0.8(\sin (1.050 t)+1.25 \sin (0.11 t+0.05))$

Eq. 1

Pitch $(y)(\mathrm{deg} / \mathrm{s} / \mathrm{s})=0.8(2.5 \sin (1.76 t+0.5)+\sin (t)-1.5$

Eq. 2

Heave $(\mathrm{G})=0.1(5 \sin (1.595 t+2)+15 \sin (1.21 t))$

Eq. 3

Surge $(G)=0.1(7.8 \sin (0.649 t+4.8)+7.8 \sin (0.825 t+3.8)+0.5)$

Eq. 4

Sway $(\mathrm{G})=0.1(18 \sin (0.583 t+5)+9 \sin (1.122 t+5.4)-0.25)$

Eq. 5 
Physiological Measurements

208 Oxygen uptake $\left(\mathrm{VO}_{2}\right)$, carbon dioxide output $\left(\mathrm{VCO}_{2}\right)$, breathing frequency $\left(f_{\mathrm{R}}\right)$ and tidal volume

$209\left(\mathrm{~V}_{\mathrm{T}}\right)$ were continuously collected by an automated breath-by-breath system (Sensor Medics ${ }^{\circledR}$

210 version Vmax ST 1.0) using a Nafion filter tube and a turbine flow meter (opto-electric).

211 Respiratory exchange ratio (RER) and minute ventilation (VE) were calculated as the quotient of

$212 \mathrm{VCO}_{2}$ on $\mathrm{VO}_{2}$ and the product of $f_{\mathrm{R}}$ and $\mathrm{V}_{\mathrm{T}}$, respectively. Heart rate values were telemetered via a

213 Polar heart rate monitor (PolarElectro, Kempele, Finland) and recorded online. Prior to testing,

214 gas analyzers and volume were calibrated with certified calibration gases $\left(16.0 \% \mathrm{O}_{2}\right.$ and $3.98 \%$

$215 \mathrm{CO}_{2}$ ) and with a 3-liter calibration syringe, respectively.

Data Reduction

218 The RMR data were truncated by 10 minutes out of 20 minutes of data collection. This

219 procedure discarded the first and last 5 minutes to nullify any metabolic rate fluctuation due to

220 familiarization with the facemask and the expectations related to the termination of data

221 collection. The metabolic data for sitting, standing, and lifting were truncated by 2 minutes out of

22210 minutes data collection to account for the metabolic inertia and depression at the beginning

223 and at the end data collection, respectively. The remaining segments (10 minutes for RMR and 8

224 minutes for the experimental conditions) were integrated and normalized over time. Oxygen

225 uptake values were converted and expressed as energy cost in metabolic equivalent (MET). The

226 same truncation and integration procedures were applied to the heart rate data. This data 
227 reduction process was undertaken to produce stable metabolic steady state information for

228 subsequent statistical analyses. Energy cost was computed using the following equation:

229 Energy cost $($ MET $)=\operatorname{VO}_{2}\left(\mathrm{ml} \mathrm{min}^{-1} \mathrm{~kg}^{-1}\right) \div 1 \operatorname{MET}\left(3.5 \mathrm{ml} \mathrm{min}{ }^{-1} \mathrm{~kg}^{-1}\right)$

231 Statistical Analyses

232 Differences between baseline, rest periods, and tasks were analyzed using repeated measures 233 analyses of co-variance (ANCOVA) with gender ( 2 levels: male and female) entered as a co-

234 variate. Post-hoc comparisons were performed using Bonferroni corrected t-tests to decompose

235 significant interaction and / or main significant effects. Assumptions of sphericity were also

236 investigated using Mauchley's test and the adjusted Greenhouse-Geisser correction factor

237 (epsilon $(\varepsilon)$ was used to identify significance $(p \leq 0.05)$ if sphericity was violated). Prior to each

238 statistical analysis, normality and homogeneity of data sets were verified via Kolmogorov-

239 Smirnov tests and Levene's tests respectively. All statistical analyses were performed using

240 Statistical Package for Social Sciences (version 21.0) (SIBM Corp., Armonk, NY, USA).

241 Results

242

243 Energy Cost

244 Results of the repeated measures ANCOVA indicated no significant differences in energy cost at

245 the beginning of each trial $(p \geq 0.05)$ suggesting participants started each trial with similar energy

246 cost. The interaction effect between motion and gender was also not significant. Analysis of

247 energy cost post-exposure revealed no significant differences in the interaction effect between

248 motion and gender for any task or motion condition $(p \geq 0.05)$. Significant differences in the main

249 effect of motion between all conditions and all tasks (Sit: $\mathrm{F}_{(1.88,0.35)}=47.67, p<0.001 ; d=0.716$,

$25095 \% \mathrm{CI}=-0.255,-0.093 ;$ Stand: $\mathrm{F}_{(1.08,26.65)}=59.16, p<0.001 ; d=0.779,95 \% \mathrm{CI}=-1.353,-0.640$; 
251 Lift: $\left.\mathrm{F}_{(1.24,18.89)}=80.67, p<0.001 ; d=0.925,95 \% \mathrm{CI}=-1.155,-0.509\right)$ (Figure 5; panel $A$ ).

252 Significant differences in energy cost were also found between NM and LM conditions but only

253 for the standing and lifting task.

254 Table 2 displays the secondary cardio-respiratory outputs $\left(\mathrm{VCO}_{2}, \mathrm{VE}, \mathrm{V}_{\mathrm{T}}, f_{\mathrm{R}}\right.$ and $\left.\mathrm{RER}\right)$ as

255 additional information; however, no statistical analyses were performed owing to the limited

256 relevance of these parameters to the research question.

257

258

259

260

261

262 Examination of the interaction effect between gender and heart rate was not statistically

263 significant for any task $(p \geq 0.05)$. Evaluation of the main effect of motion revealed statistically

264 significant differences for standing $\left(\mathrm{F}_{(1.27,759.48)}=10.14, p<0.01 ; d=0.348,95 \% \mathrm{CI}=-1.728\right.$, -

$0.806)$ and lifting $\left(\mathrm{F}_{(1.35,2274.93)}=23.10, p<0.001 ; d=0.549,95 \% \mathrm{CI}=-1.353,-0.640\right)$ but not sitting $\left(\mathrm{F}_{(1.65,6.10)}=0.50, \mathrm{p}=0.58 ; d=0.026,95 \% \mathrm{CI}=-0.279,-0.041\right)$ (Figure 5; panel B). Post hoc comparisons reveal standing in LM heart rate was significantly lower than NM ( $p=0.007$; $95 \% \mathrm{CI}=-0.664,-0.301)$ and $\mathrm{HM}(p<0.001 ; 95 \% \mathrm{CI}=-2.242,-0.896)$. Lifting heart rate during the HM condition was significantly greater than both $\mathrm{NM}(p<0.001 ; 95 \% \mathrm{CI}=-2.196,-1.040)$ and $\operatorname{LM}(p<0.001 ; 95 \% \mathrm{CI}=-1.770,-0.716)$.

271

272 Insert Figure 5 about here 


\section{Discussion}

275 Work in moving environments is perceived to be much harder than performing similar tasks in

276 stable environments. When working in moving environments fatigue can be caused by a number

277 of different factors including motion induced sickness (or perhaps the medications used to

278 mediate the effects of motion sickness), motion induced loss of sleep and motion induced

279 interruptions (due to the increased muscular effort needed to maintain postural stability (Dobbins

280 et al., 2008). However, little research has examined the fatigue related to the direct energy cost

281 due to motion-rich environments.

282

283 The use of simulated motion environments (Colwell, 2005) has provided researchers with a

284 controlled setting in which to assess human responses to motion environments and allows for

285 more controlled counterbalanced experimental designs compared to research "at sea”. While

286 research undertaken during sea trials have an element of ecological validity, a major limitation is

287 reproducibility and control of under-foot motions between experimental conditions. Developing

288 accurate performance prediction models require that experimental protocols avail of systematic

289 controls of motion-defining parameters (i.e. frequency, amplitude). The simulated motions

290 employed in this experiment were derived from sea trials and can be deemed typical of those a

291 mariner would experience, particularly on a small near-shore vessel. It was decided to increase

292 the frequency and/or amplitude profiles to increase the demands upon the participant,

293 specifically to create a distinction between the low and high motion conditions as described in

294 Table 1 (linear accelerations and angular velocities). Previous studies employed similar motion

295 characteristics as this experiment's LM profile and reflected rather calm sea conditions (Dobie

296 and May, 2002; Heus et al, 1998; Wertheim et al, 2002). The HM profile in this experiment 
297 reflects a somewhat higher sea state, with maximal linear accelerations and angular velocities at 298 least twice the magnitude of the LM profile. Roll and pitch parameters are of particular interest

299 since these types of motions have been shown to (independently or in combination) have the

300 greatest effect on MIIs and energy cost (Wertheim et al, 1994). Maximum HM roll and pitch

301 components were $141 \%$ and $191 \%$ larger than the LM condition's, respectively, therefore,

302 showing that an increase in the magnitude of the sea-state will result in increased energy cost.

303 These profiles are likely more reflective of larger vessel and more deep-sea locations.

305 Results of this study indicate that seafarers may have significantly increased energy cost due to

306 working in motion-rich environments. Thus, long-term exposure to motion-rich environments

307 will likely have a cumulative fatigue effect that can potentially lead to increased risk of falls,

308 musculoskeletal injury, and human error. This is especially problematic in the marine context

309 since workers can be exposed to vessel motions for extended periods of time, increasing the risk

310 of the negative effects of motion-induced fatigue (Baitis et al, 1995; Colwell, 1989). The focus of

311 this paper was to quantify energy cost of persons performing three common tasks (sitting,

312 standing and $\mathrm{MMH}$ ) during three different motion conditions. Results indicate significant

313 differences in energy cost between all motion conditions for nearly all tasks (all motion

314 conditions were significantly greater than NM while HM was significantly greater than LM).

315 Findings show that as motion intensity increases, so does energy demand and these outcomes fit

316 with previous examinations of $\mathrm{O}_{2}$ uptake and energy cost in moving environments (Heus et al.,

317 1998; Wertheim et al., 2002; Breidahl et al., 2013). However, others have reported that walking

318 on a laterally oscillating platform can reduce energy cost of walking (Joshi V et al. 2015). These

319 outcomes tend to show that humans may not be able to entrain to it. Nevertheless, the current 
article operates far from such entrainment regimes, thereby generally resulting in increased

energy cost. In other words, there should not always be an increase in energy costs on a moving

322 platform.

323

324 While statistical analyses between tasks (i.e. sitting, standing and $\mathrm{MMH}$ ) may be of interest,

325 intuitively it is understood there will be differences in selected tasks' workload demands. Energy

326 requirements will increase proportionately with increased workload (Astrand and Rodahl, 1986).

327 Comparing the three tasks, energy requirements were lowest for the sitting task, followed by

328 standing, and highest for lifting/lowering (see Figure 3, panel A). This seems reasonable given

329 the potential increased instability of upright bipedal stance compared with sitting in a chair with

330 back support.

331

332 NM sitting energy cost values were comparable to previous studies (Astrand and Rodahl, 1986;

333 Levine et al, 2000). There was a significant (25\%) increase in the HM sitting energy cost and a

334 significant (5\%) increase in the LM sitting condition. These differences were considerably less

335 than the standing and lifting tasks. These findings seem reasonable because during sitting the

336 participant's CoM likely remains over the base of support, predominantly defined by the area of

337 the seat pan and foot positions on the floor (i.e. larger base of support). No correction for loss of

338 balance are required and it is likely that the majority of any additional muscular activation relates

339 to trunk stabilization and may primarily be related to only head/upper torso stabilization. Sitting

340 energy cost may be most affected by motions involving increased forward pitch velocities since

341 operators can rely on the backrest for high backward pitch moments. Roll motions would have a

342 lesser effect on energy demands if arm rests are present since arm stabilization will mediate side- 
343 to-side movements. Even though relatively small sitting energy cost changes were observed

344 across motions conditions, long-term exposure to motions could still induce a fatiguing effect.

345

346 NM standing energy cost was also consistent with previous findings (Garg et al, 1978; Houdijk

347 et al, 2009; Pandolf et al, 1977; Levine et al, 2000). Differences in NM energy cost were much

348 more apparent during standing trials, with increases of $34.7 \%$ and $157.7 \%$ for LM and HM

349 conditions, respectively. Unlike sitting, standing creates situations for reduced postural stability

350 and thus greater mechanical demands upon the operator. While standing in motion rich

351 environments workers are exposed to greater motion-induced moments of inertia that directly

352 influence an operator's ability to maintain the centre of mass (COM) within BoS limits. When

353 the CoM reaches or exceeds the functional stability limits, balance is compromised, and

354 operators have no option other than employing postural adjustments to correct for balance

355 perturbations (Winter, 1995). Standing in high motion states will require the operator to

356 continually react and correct for perturbations. Previous work by Duncan et al. (2007) has found

357 that as platform perturbation magnitudes increase so do the number of stepping strategies that are

358 used to maintain balance. This increasing magnitude of postural responses would require greater

359 energy cost due to the increased muscular contractions required to move the limbs. Additionally,

360 when not stepping to regain balance, the muscular contractions required to produce a moment to

361 keep the CoM within the BoS to retain balance in response to larger perturbations would also be

362 greater, and thus, requiring a higher energy cost.

363

364 Interestingly, the impact of motion on heart rate and energy cost was different. While significant

365 changes in energy cost were displayed for all tasks, only the lifting task displayed a similar 
366 increasing trend with increased motion, while heart rate during LM was significantly less than

367 both high and no motion conditions. This is unlike previous findings (Heus et al,1998) that found

368 the energy costs were accompanied by increases in heart rate; however, in their study only one

369 motion condition was measured in both seas and simulated ocean conditions. It is plausible that

370 during LM positive inotropic agents (e.g., catecholamines) increased ventricular contraction and,

371 therefore, systolic ejection volume to maintain tissue oxygen perfusion at the cellular-capillary

372 level. Future research is needed to confirm these hypotheses.

373

374 While this study examined only two motions in comparison to a baseline (no motion) condition,

375 future work should examine a broad spectrum of motions described by systematic changes in

376 frequency and amplitude in all 6 degrees of freedom. Such an experimental approach will allow

377 for regression analyses to be used to describe platform motions and task-specific energy cost.

378 Increased energy cost likely results in increased fatigue of the maritime worker, and in turn, may

379 cause decreased ability to perform work effectively (or safely). Additionally, general fatigue may

380 result in decreased morale of workers as the effects of motion-induced fatigue are typically

381 considered a negative subjective experience (Fu et al., 2001; Shen et al., 2006). Acute and

382 chronic fatigue appears to be dependent on a number of factors specific to a seafaring occupation

383 including tour length, environmental factors, extended shift length and switching from sea to port

384 with fatigue appearing to increase at the end of tours (Wadsworth, 2006; Wadsworth et al.,

385 2008). This may lead to increased risk of injuries and errors that could lead to further accidents

386 and injuries. 
388 More research effort must focus on the mechanisms and outcomes of motion-induced fatigue. A

389 better understanding of motion-induced fatigue will allow for improved planning of work for

390 persons at sea for extended periods of time. While this study demonstrated increases in energy

391 cost over a short period of time, this may or may not reflect extended exposure time responses.

392 Defining the relationship between platform motions and task-specific energy demands will

393 contribute to developing guidance notes and strategies regarding habitability, work-rest ratios

394 and shift work scheduling, accidents and injuries, crewing and crew satisfaction, nutrition

395 demands and workstation and vessel design.

396 Ergonomic Application.

397 Ergonomists and human factors engineers must consider these increased energy costs when

398 evaluating occupational demands of seafarers. Workers in moving environments require more

399 energy to perform equivalent tasks to those working in non-moving environments. Therefore,

400 manual materials handling guidelines, for example, must be sensitive to the environment the task

401 occurs. For example, currently the NIOSH lifting equation, assuming the baseline maximum

402 aerobic capacity of U.S. workers is $9.5 \mathrm{kcal} \mathrm{min}^{-1}$ (aerobic lifting capacity of an average 40-year

403 old female worker), workers should be lifting at no more than $50 \%$ of their maximum (4.75 kcal

$\left.404 \mathrm{~min}^{-1}\right)$ for 1 hour or less, $40 \%$ of their maximum $\left(3.8 \mathrm{kcal} \mathrm{min} \mathrm{m}^{-1}\right)$ for $1-2$ hours; and $33 \%$ of their

405 maximum (3.1 kcal min-1) for 2-8 hours (Waters et al., 1993). Conversion of the results of this

406 study to $\mathrm{kcal} \mathrm{min}^{-1}$ has found that during the LM and HM condition the energy cost is 3.78 and

$4075.33 \mathrm{kcal} \mathrm{min}^{-1}$, respectively. Therefore, changes to the lifting task would have to be made in

408 order to make it safe for workers performing this same task in a moving environment. Based on

409 the increases in energy cost during the standing and holding tasks, more conservative estimates

410 would also need to be applied to all MMH tasks, including pulling pushing and carrying. 


\section{Conclusions}

413 This research reflects attempts to develop an experimental protocol to assess the effects of

414 motion on the energy demands of persons working in motion-rich environments.

415 From the results, it can be concluded:

416 1. Energy cost of common manual materials handling tasks are greater in moving

417 environments when compared to non-moving environments.

418 2. Energy cost demands are dependent upon the magnitude of the platform perturbations

419 and the nature of the task being performed, with tasks that involve the stabilization of 420 upright stance having higher energy demands.

3. These results provide objective outcomes on energy cost of commonly performed tasks study outcomes to mitigate motion-induced fatigue with the aim to maintain operator performance, minimize human factors errors, and reduce work-related injuries.

\section{Acknowledgements}

428 The authors would like to thank the participants for their valuable cooperation to the study. We 429 would like also to thank Dr. Thamir Alkanani for his assistance. 


\section{References}

432 Astrand, P. and Rodahl, K. (1986). Textbook of Work Physiology. McGraw-Hill, New York, 433 USA.

434 Baitis, A. E., Holcombe, F. D., Conwell, S. L., Crossland, P., Colwell, J., Pattison, J. and Strong, R. (1995). 1991- 1992 Motion Induced Interruptions (MII) and Motion Induced Fatigue (MIF) Experiments at the Naval Biodynamics Laboratory. Technical report for the

Bles, W., Bos, J.E., de Graff, B., Groen, E. and Wertheim, A.H.(1998). Motion sickness: Only one provocative conflict? Brain Research Bulletin, 47(5): 481-487.

Breidahl, T., Christensen, M., Jepsen, J. R., Johansen, J. P., and Omland, Ø. (2013). The influence of ship movements on the energy expenditure of fishermen: A study during a North Sea voyage in calm weather. International Maritime Health, 64(3), 114-120.

Colwell, J. L. (1989). Human Factors in the Naval Environment: A Review of Motion Sickness and Biodynamic Problems. DREA Technical Memorandum 89/220, Canadian National Defence Research Establishment Atlantic, Dartmouth. Group. (2006). Best practice methods to apply to measurement of resting metabolic rate in adults: a systematic review. Journal of the American Dietetic Association, 106(6), 881903. 
451 Crossland, P. (1994). Experiments to quantify the effects of ship motions on crew task performance - Phase II, Assessment of Cognitive performances (Rep. No. Technical report DRA/AW/AWH/TR94001, Defense Research Agency, Farnborough, UK.).

454

456
Crossland, P. and Lloyd, A. R. J. M. (1993). Experiments to quantify the effects of ship motions on crew task performance -Phase I, motion induced interruptions and motion induced fatigue (Rep. No. Technical Report DRA/AWMH/TR93025, Defense Research Agency, Farnborough, UK).

Dobbins, T., Rowley, I., Campbell, L., 2008: High Speed Craft Human Factors Engineering Design Guide. ABCD-TR-08-01 V1.0.

Dobie, T.G. (2003). Critical Significance of Human Factors in Ship Design. Proceedings of the 2003 RVOC Meeting, Large Lakes Observatory, University of Minnesota, 8 - 10 October, 2003.

Dobie, T.G. and May, J.G. (2002). Effects of fatigue and motion sickness on naval task performance. Naval Biodynamics Laboratory, University of New Orleans, New Orleans, USA.

Duncan, C. A., Albert, W. J., Langlois, R. G., \& MacKinnon, S. N. (2014). Stepping response during constrained and unconstrained standing in moving environments. International Journal of Maritime Engineering, 156, 207-212.

Duncan, C. A., Mansfield, A., Byrne, J. M., Ingram, T. McIlroy, W. E. (2016). Populations differences in postural response strategy development associated with continuous 
471

472

473

474

475

476

477

478

479

480

481

482

483

484

485

486

487

488

489

490

perturbation stimuli: Would dancers have better balance on a boat? PlosOne 11(11), $\mathrm{e} 0165735$.

Duncan, C. A., MacKinnon, S. N., Albert, W. J., Antle, D. M., and Matthews, J. (2007). Effects of simulated vessel motions on thoracolumbar and centre of pressure kinematics. Occupational Ergonomics, 7(4), 265-274.

Duncan, C. A., MacKinnon, S. N., and Albert, W. J. (2010). Changes in thoracolumbar kinematics and centre of pressure when performing stationary tasks in moving environments. International Journal of Industrial Ergonomics, 40, 648-654.

Duncan, C. A., Langlois, R. G., Albert, W. J., and MacKinnon, S. N. (2014). The habituation of human postural responses to platform perturbations. International Journal of Industrial Ergonomics, 44(6), 874-881.

Duncan, C. A., MacKinnon, S. N., and Albert, W. J. (2012). The effects of moving environments on thoracolumbar kinematics and foot centre of pressure when performing lifting and lowering tasks. Journal of Applied Biomechanics, 28(2), 111-119.

Garg, A., Chaffin, D.B., and Herrin, G.D. (1978). Prediction of metabolic rates for manual materials handling jobs. The American Industrial Hygiene Association Journal, 39(8), 661-674.

Haward, B.M., Lewis, C.H., and Griffin, M.J. (2009). Motions and crew response on an offshore oil production and storage vessel. Applied Ergonomics, 40, 904-914. 
491 Heus, R., Wertheim, A.H., and Havenith, G. (1998). Human energy cost when walking on a moving platform. European Journal of Applied Physiology, 7, 388-394.

493 494

Houdijk, H., Fickert, R., van Vetzen, J., and van Bennekom, C. (2009). The energy cost for balance control during upright standing. Gait and Posture, 30, 150-154.

Joshi, V. and Srinivasan, M. (2015). Walking on a moving surface: energy-optimal walking motions on a shaky bridge and a shaking treadmill can reduce energy costs below normal. Proceedings of the Royal Society A: Mathematical, Physical, and Engineering Sciences, 471, 20140662. DOI: 10.1098/rspa.2014.0662.

Levine, J.A., Schleusner, S.J. and Jensen, M.D. (2000). Energy cost of non-exercise activity. American Journal of Clinical Nutrition, 72(6), 1451-1454.

Lloyd, A.R.J.M. Seakeeping: Ship Behaviour in Rough Weather. 1993. RINA, London.

MacKinnon, S.N., Matthews, J., Holmes, M. and Albert, W.J. (2011). The effect of platform motions upon the biomechanical demands of lifting tasks. Occupational Ergonomics, 10, 103-112.

Pandolf, K.B., Givoni, B., and Goldman, R.F. (1977). Predicting energy-cost with loads while standing or walking very slowly. Journal of Applied Physiology, 43(4), 577-581.

Stevens, S. and Parsons, M. (2002). Effects of motion at sea on crew performance: A survey. Maritime Technology, 39(1), 29-47.

Shen, J., Barber, J., and Shapiro, C.M. (2006). Distinguishing sleepiness and fatigue: Focus on definition and measurement. Sleep Medical Reviews, 10(1), 63-76. 
511 Wadsworth, E. J., Allen, P. H., Wellens, B. T., McNamara, R. L., and Smith, A. P. (2006).

512 Patterns of fatigue among seafarers during a tour of duty. American Journal of Industrial

$513 \quad$ Medicine, 49(10), 836-844.

514

515 Wadsworth, E. J. K., Allen, P. H., McNamara, R. L., and Smith, A. P. (2008). Fatigue and health

516 in a seafaring population. Occupational Medicine, 58, 198-204.

517 Waters, T. R., Putz-Anderson, V., Garg, A., \& Fine, L. J. (1993). Revised NIOSH equation for 518 the design and evaluation of manual lifting tasks. Ergonomics, 36(7), 749-776.

519 Wertheim, A. (1998). Working in a Moving Environment. Ergonomics, 41(12), 1845 - 1858.

520 Wertheim, A. H., Bos, JE., and Bles, W. (1998). Contributions of roll and pitch to seasickness. Brain Research Bulletin 47(5), 517-524.

522 Wertheim, A.H., Heus, R. and Vrijkotte, T.G.M.(1994). Energy Cost, Physical Workload and Postural Control During Walking on a Moving Platform. Defense Report TD 94-0048, TNO Institute for Human Factors, Soesterberg, Netherlands.

525

526

527

528

529

Wertheim, A. H., Kemper, H. C. G., and Heus, R. (2002). Maximal oxygen uptake during cycling is reduced in moving environments; consequences for motion-induced fatigue. Ergonomics, 45(3), 186-202.

Winter, D.A. (1995). Human balance and posture control during standing and walking. Gait and Posture, 3(4), 193-214. 


\section{Table $\mathbf{1}$ (on next page)}

Motion profile characteristics including maximum, minimum and root mean square of each degree of freedom for low and high motion conditions. 
1 Table 1: Motion profile characteristics including maximum, minimum and root mean square of

2 each degree of freedom for low and high motion conditions.

\begin{tabular}{lcccccc} 
Degree of & \multicolumn{3}{c}{ Low Motion } & \multicolumn{3}{c}{ High Motion } \\
Freedom & RMS & Max. & Min. & RMS & Max. & Min. \\
\hline Sway (g) & 0.11 & 0.22 & -0.22 & 0.12 & 0.24 & -0.24 \\
Surge (g) & 0.23 & 0.44 & -0.44 & 0.25 & 0.48 & -0.48 \\
Heave (g) & 0.24 & 0.43 & 0.00 & 0.26 & 0.47 & 0.00 \\
Pitch (deg/s/s) & 3.74 & 5.30 & -5.30 & 10.24 & 14.51 & -14.50 \\
Roll (deg/s/s) & 4.42 & 6.99 & -6.99 & 11.97 & 16.97 & -16.97 \\
Yaw (deg/s/s) & 0.00 & 0.00 & 0.00 & 0.00 & 0.00 & 0.00 \\
\hline
\end{tabular}

3 


\section{Table 2 (on next page)}

Cardiorespiratory parameters recoded during the experimental sessions with the indirect calorimetry system.

Mean (SD) 
Table 2: Cardiorespiratory parameters recoded during the experimental sessions with the indirect calorimetry system

2

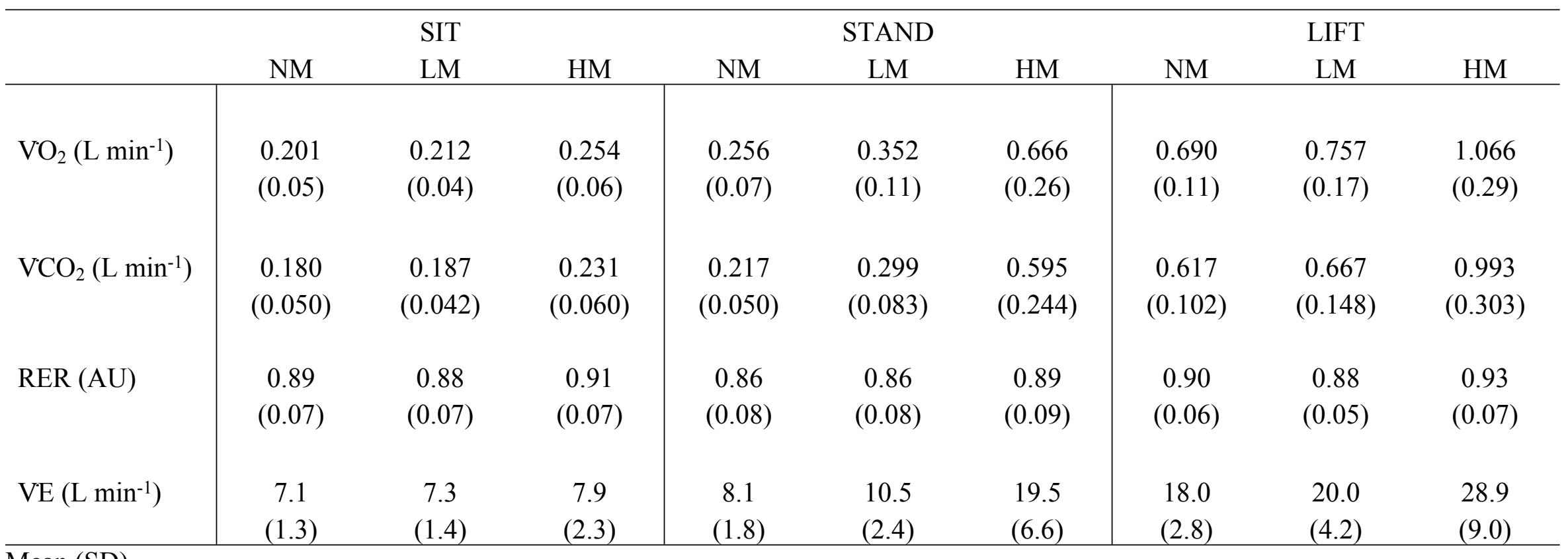

3 Mean (SD) 
Figure 1

Experimental design schematic

Participants to perform a task (i.e. quiet sitting on a chair, standing in an upright position, or lifting and lowering a load) for each of the three motion conditions. Each session (NM, LM, $\mathrm{HM}$ ) began with quiet sitting while resting metabolic rate (RMR) was collected. Exposure to each motion condition lasted ten minutes with a minimum of a five-minute rest period between conditions. The no motion trial was always presented to the participant first.

\begin{tabular}{|c|c|c|c|}
\hline $\begin{array}{c}\text { No Motion } \\
\text { (Sit, Stand, or lift) } \\
10 \text { min }\end{array}$ & $\begin{array}{c}\text { Motion 1 } \\
5 \text { min recovery }\end{array}$ & $\begin{array}{c}\text { Motion 2 } \\
\text { (Sit, Stand, or lift) } \\
10 \text { min }\end{array}$ & $\begin{array}{c}5 \text { min recovery } \\
\text { (Sit, Stand, or lift) } \\
10 \text { min }\end{array}$ \\
\hline
\end{tabular}




\section{Figure 2}

Motion platform setup depicting standing.

All motion conditions were performed on a Moog 6DOF2000E electric motion platform. The platform consisted of a 2 by $2 \mathrm{~m}$ metal platform with $1.02 \mathrm{~m}$ high railings along the perimeter. A canopy enclosure eliminated external horizontal and vertical cues from the participant's field of vision. Motion conditions varied in amplitude and frequency and were derived from captured wave induced ship motions using linear wave theory that allowed for the profile to vary in magnitude (Lloyd, 1993). Photo credit: Carolyn A. Duncan. 


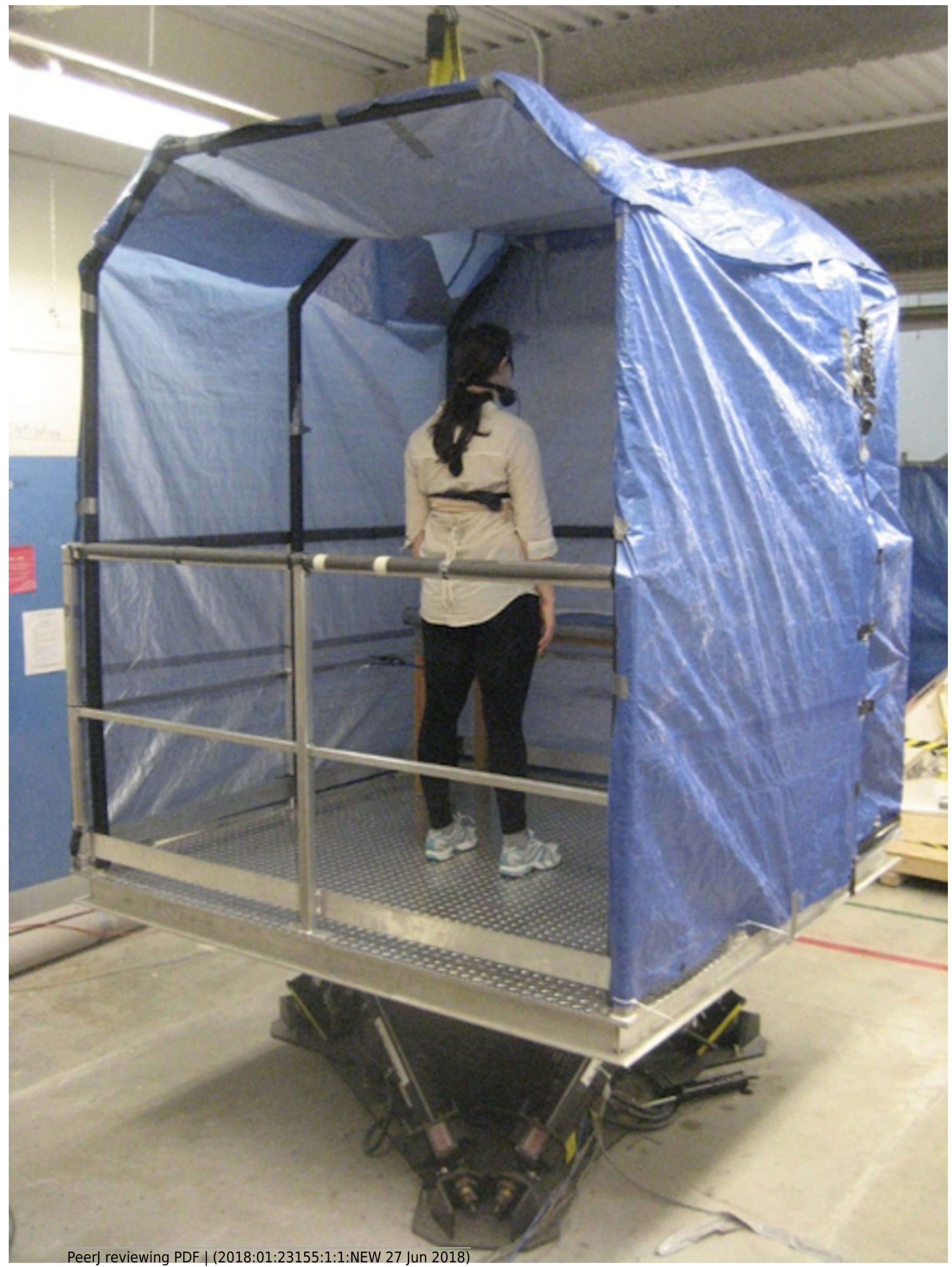




\section{Figure 3}

Sagittal view of the lifting task that participants performed on the motion platform.

A bimanual sagittal plane lifting/lowering task required participants to lift and lower a $5.0 \mathrm{~kg}$ load (length: $0.327 \mathrm{~m}$; width: $0.327 \mathrm{~m}$, height: $0.270 \mathrm{~m}$ ) directly to and from a table (width: $1.54 \mathrm{~m}$, depth: $0.52 \mathrm{~m}$; height from floor: $0.72 \mathrm{~m}$ ) securely situated $0.60 \mathrm{~m}$ directly in front of the participant facing the bow direction. Photo credit: Carolyn A. Duncan.

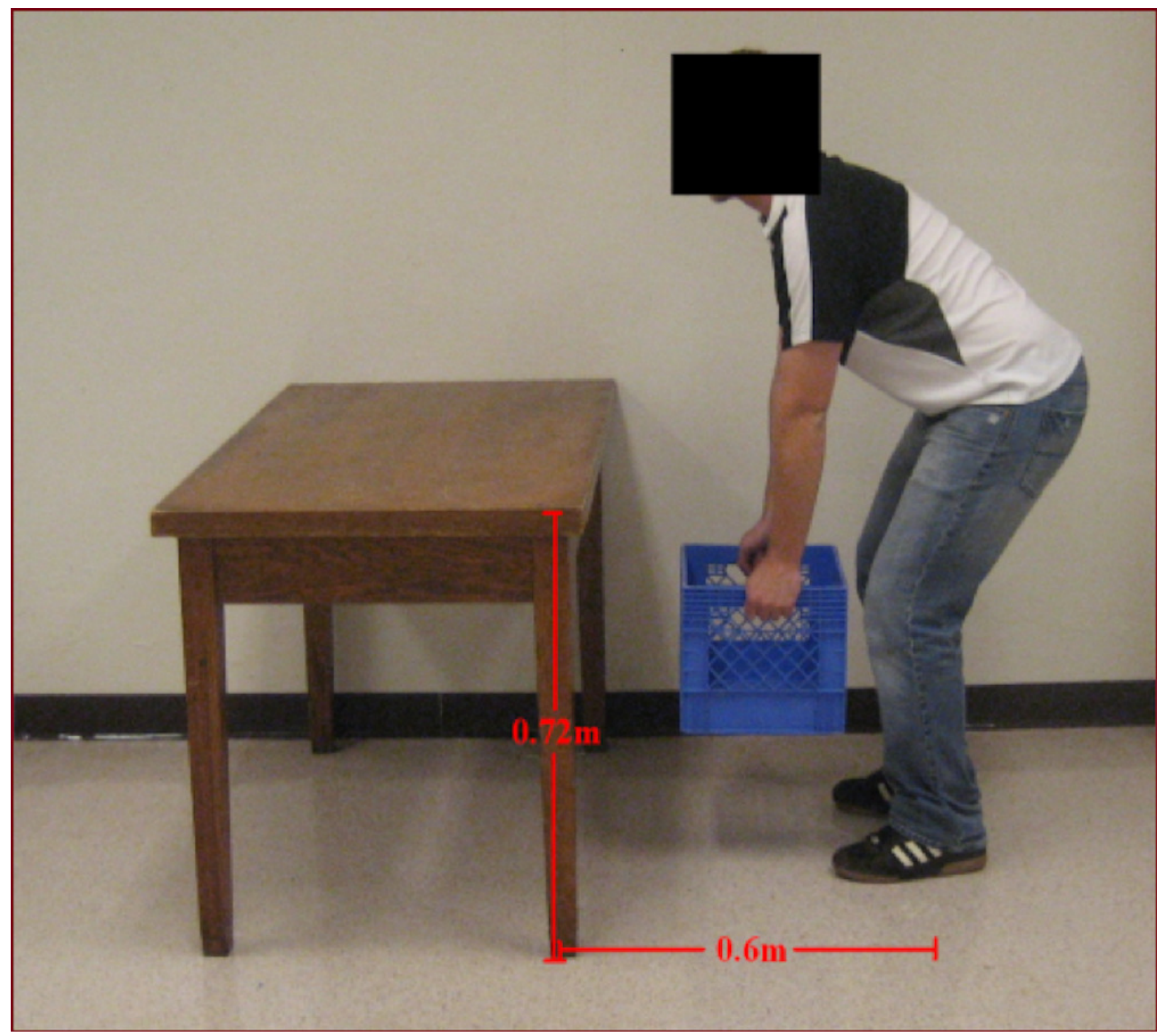


Figure 4

Schematic of 5 degree of freedom ship motions.

Linear equations used to develop all motion profiles are detailed (Equations 1-5). The low motion (LM) profile reflected a $10 \%$ increases in motion frequency relative to the original vessel from which the motion profiles were recorded and the high motion (HM) profile was characterized by a $15 \%$ increase in frequency and a $265 \%$ increase in amplitude relative to the seagoing motion profile.
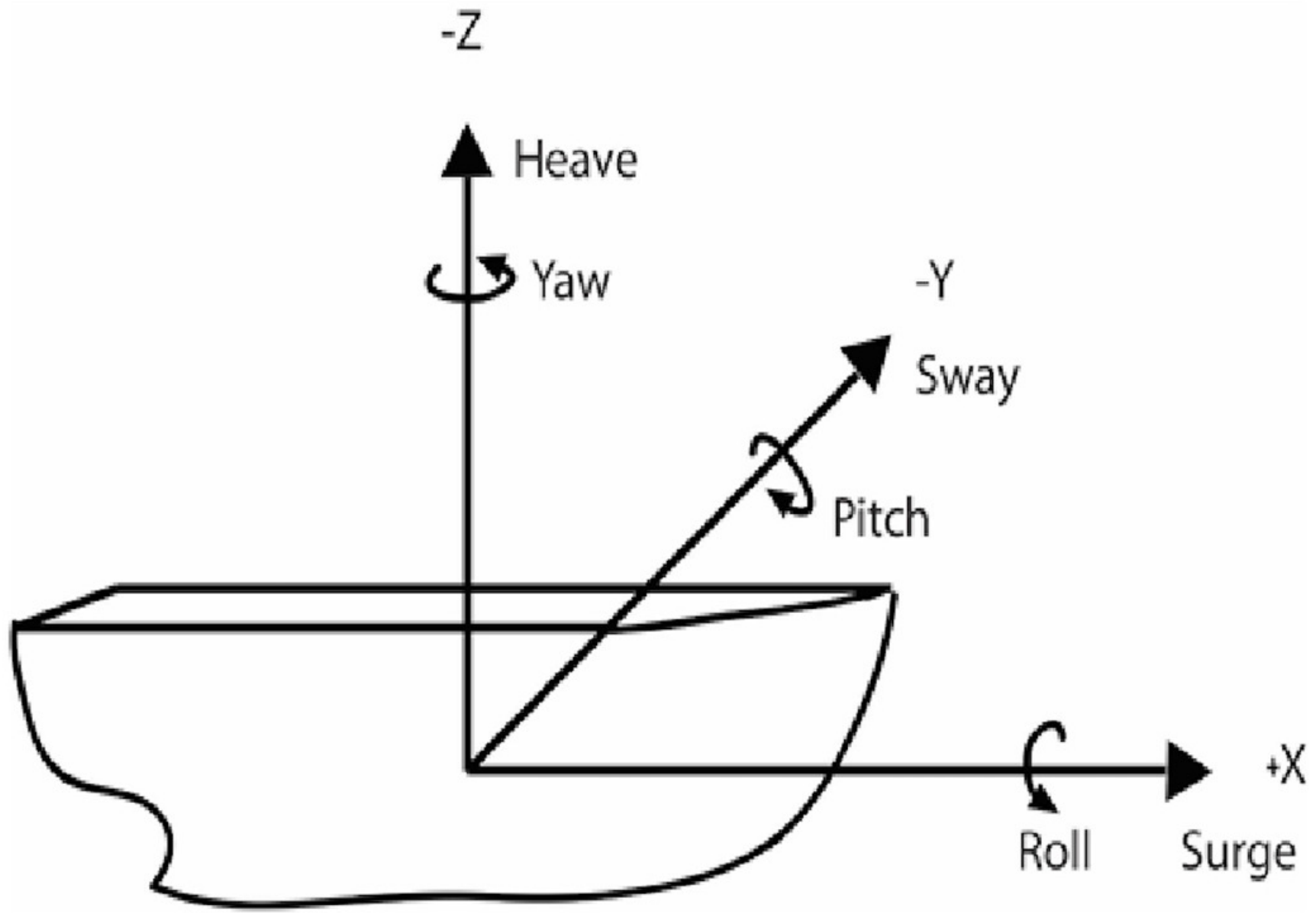


\section{Figure 5}

Energy cost of lifting and HR responses during experimental conditions.

Panel A - A comparison of mean energy cost (MET) and corresponding standard deviations between motion conditions for all tasks. Statistical significant difference $(p<0.05)$ from No Motion $(*)$ and from other two motion conditions (**). Panel B -A comparison of mean energy cost (MET) and corresponding standard deviations between motion conditions for all tasks. Statistical significant difference $(p<0.05)$ from two motion conditions $(* *)$. 

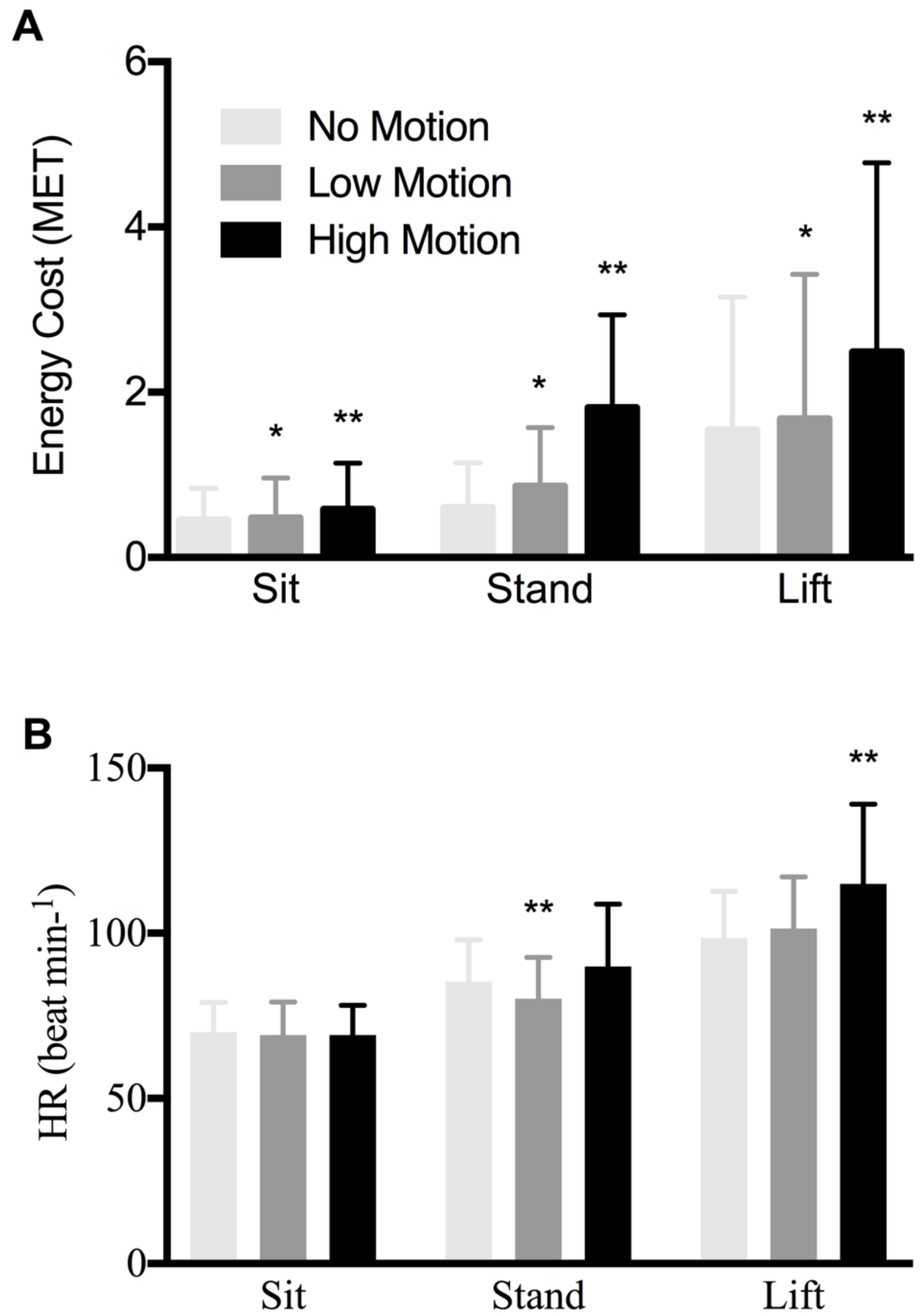